\title{
Evolutionary optimization of reinforced concrete beams, taking into account design reliability, safety and risks during the emergency loss of supports
}

\author{
Ashot Tamrazyan and Anatoliy Alekseytsev* \\ Moscow State University of Civil Engineering, Yaroslavskoe shosse, 26, Moscow, 129337, Russia
}

\begin{abstract}
An algorithm has been developed to optimize the reinforced concrete beams in removing the supports based on an adapted genetic algorithm and RBDO approach. Multiple cross-sectional dimensions of elements, concrete class, class and diameter of the reinforcements vary. Avoiding significant changes in the geometry of the structure after emergency actions is considered as the main active constraints. It is believed that this constraints provides the required structural resistibility in an emergency situation. The value terms of material losses risk in case of a possible structure failure as a component of the objective function is considered. The proposed genetic algorithm includes an adapted mutation operator and the elitism strategy for selecting the best solutions. The algorithm presented allows obtaining design solutions of high operational reliability for rectangular reinforced concrete beams, taking into account the optimal ratio of a structure costs and the risks of its failure in an accident.
\end{abstract}

\section{Introduction}

Investigation of structural systems resistibility under emergency actions is one of the current trends in the construction science [1-4]. Both local damage in the form of removing supports $[5,6]$, temperature and fire $[7,8]$ effects, corrosion damage [9], impacts $[10,11]$ and other natural and man-made factors are considered.

Requirements to prevent the destruction of the structure at least for some time usually lead to a significant increase in object cost. Therefore, the development of optimal design methods taking into account the emergency actions is a very important task. At the same time, it is necessary to minimize material losses. This is possible when assessing an emergency risk and considering this risk directly in the process of optimal structure designing. As for reinforced concrete structures, low attention span is paid to such optimization methods in the literature.

\footnotetext{
*Corresponding author: aalexw@mail.ru
} 
The increasing complexity of buildings leads to an increase in the complexity of bearing capacity systems designing. Therefore, the development and improvement of automated calculation and optimization algorithms is required. This also increases the requirements for a number of variable parameters, which grows year by year. Today it seems necessary to perform a search on the design parameters defined by sets of discrete values, which fully reflects the features of solution synthesis for complex structures. When solving such tasks with a relatively large number of variable parameters, evolutionary modeling has increasingly been used [12-16]. This article proposes one of such methods.

\section{Formulation of the optimization problem}

Reinforced concrete beams with steel reinforcement without pre-stressing and having resistance to damage in an accident (with increased survivability) are considered. The cross-section of a beam is assumed constant on length. The arrangement of reinforcement location in cross section does not vary. As the optimal design goal, it is proposed to achieve the value of relative risk of possible material losses, associated with the failure of the structure, the specified safety level indicators:

$$
R=r / C \rightarrow \Omega_{l} \in\{\Omega\} ; \quad C=f\left(C_{b}\left(\left\{y_{b}\right\},\left\{G_{b}\right\}\right)+C_{a}\left(\left\{y_{a}\right\},\left\{D_{a}\right\}\right)\right)
$$

where $C$ is the structure conditional cost value, $r$ is a total potential damage from the structure destruction in a building, expressed in structure conditional cost value units; $\Omega_{l}$ is the safety level, assigned by the designer with regard to ensuring reliability, it is a real number, which is recommended to be assigned in the interval $0.05-1 ; l \in[1 . . L] ;\{\Omega\}$ is a set of safety levels with the number of components $L ; C_{b}\left(y_{b}, G_{b}\right)$ is the cost of concrete determined by sets of $y_{b}$ values of its class and a set of $G_{b}$ parameters of its section geometry; $C_{a}\left(\left\{y_{a}\right\},\left\{D_{a}\right\}\right)$ is the cost of reinforcement depending on the values of $y_{a}$ set of resistances typical for its class and the values of $D_{a}$ set of its diameters groups. All these sets will be represented in the form of ordered pairs, sorted by descending characteristic values.

Set

$$
\left\{y_{b}\right\}=\left\{\left(R_{b 1}, R_{b t 1}\right),\left(R_{b 2}, R_{b t 2}\right), \ldots,\left(R_{b N}, R_{b t N}\right)\right\},
$$

where $R_{b 1}-R_{b N}, R_{b t 1}-R_{b t N}$ is design concrete resistances to compression and tension, respectively, $N$ - the number of concrete classes allowed for selection in solving the tasks.

Set

$$
\left\{G_{b}\right\}=\left\{\left(\left\{h_{1}\right\},\left\{b_{1}\right\}\right)_{1},\left(\left\{h_{2}\right\},\left\{b_{2}\right\}\right)_{2}, \ldots,\left(\left\{h_{m}\right\},\left\{b_{m}\right\}\right)_{m}\right\},
$$

where $h, b$ is vertical and horizontal section size, $m$ - the number of cross section sizes allowed for selection. Set sizes $\{h\},\{b\}$ are determined by cross-section shape. While for a rectangular section, they are determined by height and width, respectively. For more complex sections, the number of set sizes for the element in $\left\{G_{b}\right\}$ set will increase. For example, for a T-section, it will be two sizes in height, the sum of which determines the overall height of the section, and two sizes in width: the width of the shelf and the wall.

The sets $D_{a}, y_{a}$ will be represented as follows: 


$$
\left\{D_{a}\right\}=\left\{\left(d_{1} . . d_{n s 1}\right)_{R s 1},\left(d_{1} . . d_{n s 2}\right)_{R s 2},\left(d_{1} . . d_{n s N a}\right)_{R s N a}\right\},\left\{y_{a}\right\}=\left\{R_{s 1}, R_{s 2}, \ldots, R_{s N a}\right\}
$$

where $\left(d_{1} . . d_{n s 1}\right)_{R s 1}$ is diameters of class 1 reinforcement with resistance $R_{s 1}$, the number of which is chosen to be equal $n s 1$ in course of searching a solution. The variable $R_{s}$ is the design reinforcement tension stress resistance, $N_{a}$ - number of reinforcement classes.

In accordance with the provision of the condition of the structure resistability to progressive destruction (survivability conditions), we will set a limit on $f$ structure displacements that should not exceed the allowable values $[f]$ :

$$
f \leq[f]=\left\{\begin{array}{l}
0,3 H,(2,5 \leq \mathrm{H} \leq 3,2) \\
0,5 H,(2,5 \leq \mathrm{H} \leq 3,2)
\end{array},\right.
$$

where $H$ is the height from floor deck to the bottom face of the structure (m).

\section{Optimization problem solution method}

Perform the following key steps.

1. Setting initial data on the design object. At this stage, the set of values of the construction parameters necessary for its calculation is determined (loads are collected, materials constants and support fixings are set), and discrete sets of variable parameters are formed. The structure variant will be represented in the form of such a data structure $\tilde{O}=[R ; \vec{P}]$, where $R$ is the value of the objective function for the object (1), $\vec{P}$ is vector of values of variable parameters. We will form two sets of design options (fig.1, a, b), where $N g$ is number of $\tilde{O}$ objects in groups considered at each iteration. This value must be a multiple of four. Initially these sets are empty.

2. Iteration process.

2.1 Formation of set (I) (fig. 1,a). In the algorithm operation there are 2 cases: when set (II) (fig. 1,b) is empty and when it's not. In the first case, the generation of $\mathrm{Ng} / 4$ variants of structure is executed according to the principle of decreasing of parameter values in vector $\vec{P}$. Thus, we guarantee that at least a few structure versions will pertain to the feasible region (if the initial sets of variable parameters are correctly formed). The rest $3 \mathrm{Ng} / 4$ variants of structures are formed by random selection of parameters in their acceptable region. In second case, set (I) is formed as follows: $3 \mathrm{Ng} / 4$ objects are added to set (I) from set (II) and modified by the genetic mutation operator, while the remaining options are generated randomly. We will consider the mutation operator let three parameters $A, B, C$ vary. Each parameter has a discrete set of values allowed for selection.

$$
A:\left\{a_{1}, a_{2}, a_{3}, a_{4}, a_{5}\right\}, B:\left\{b_{1}, b_{2}, b_{3}\right\}, C:\left\{c_{1}, c_{2}, c_{3}, c_{4}, c_{5}, c_{6}, c_{7}\right\}
$$

Is introduced the integer $k$, determining the relative distance in the line from one parameter value to another. In the theory of genetic algorithms, this distance is called the Hamming distance. At this rate, $k\left(c_{1} c_{2}\right)=k\left(c_{1} c_{2}\right)=\ldots .=k\left(c_{6} c_{7}\right)=1, k\left(c_{5} c_{7}\right)=2$, $k\left(c_{2} c_{7}\right)=5$, for the first numbers of values to the direction of decreasing the positions in line $k=0$, as for the last positions in the increasing direction etc. 


$$
\begin{aligned}
& \tilde{O}_{1 \ni}=\left[C_{1 \ni} ; \vec{P}_{1 \ni}\right] \\
& \tilde{O}_{2 \ni}=\left[C_{2 \ni} ; \vec{P}_{2 \ni}\right] \\
& O_{N g \ni}=\left[C_{N g \ni} ; \vec{P}_{N g \ni}\right]
\end{aligned}
$$

a) b)

\section{Example of mutation operator's work}

$$
\begin{gathered}
M\left|\vec{P}_{1}\right|=M\left|\left\{a_{3}, b_{1}, c_{4}\right\}\right|=\{\tilde{\vec{P}}\}= \\
=\left\{\begin{array}{c}
\left\{a_{3}, b_{2}, c_{2}\right\},\left\{a_{3}, b_{3}, c_{2}\right\},\left\{a_{3}, b_{2}, c_{3}\right\} \\
\left\{a_{3}, b_{3}, c_{3}\right\},\left\{a_{3}, b_{2}, c_{5}\right\},\left\{a_{3}, b_{3}, c_{5}\right\} \\
\left\{a_{3}, b_{2}, c_{6}\right\},\left\{a_{3}, b_{3}, c_{6}\right\}
\end{array}\right\}
\end{gathered}
$$

c)

Fig. 1. Groups of versions of objects in iteration (a),(b); Demonstrating the mutation operator's work (c)

In this case, we will perform random changes taking into account the Hamming distance, which reference direction is also chosen randomly. As we approach the optimum, the $k$ value in parameter changing is taken equal to one or two, depending on the location in the set of the current parameter value. Then as for the parameter $a_{2}(6)$, with the direction from left to right, this distance may take the value 2 , and with the direction from right to left, $k=1$. The criterion of approaching the optimum will be considered the degree of changing the objective function $R$ depending on a change in the parameter values. In changing $R$ value by less than $20 \%$ the number of the parameters varied decreases to 1 , while $k_{\max }=2$. If the actual distance $r<2$, then $k=1$ and changes are made in any of the directions available. If $R$ varies within $20-50 \%$ then no limits are applied to random changes. We will provide the operator's work result in varying the parameters $B, C$ under the condition of proximity to the extremum. We will write down possible variants of resulting vectors $\tilde{\vec{P}}_{1}$ for initial vector of $\vec{P}_{1}$ parameters, denoting the mutation operator as $M|\bullet|($ fir. 1,c).

2.2 Calculation of the stress - strain state of the structure and its initial design reliability. The mathematical expression described in SP 63.13330.2012, SP 52-103-2007 as a tool for calculating a structure variant in the search for a rational solution are used. Herewith, we take into account the nonlinearity associated with the formation of cracks and concrete creep by lowering the initial elasticity modulus on certain parts of the structure. In this case, first, a calculation is made for the defining stressing, during which these sections are determined, and then recalculation is performed in the internal iteration cycle, taking into account the reduced elastic modulus. The dynamic effect associated with the rapid removal of supports from the design scheme is broadly considered based on G.A. Geniev's energy approach. According to this method, the dynamic force value $T_{D}$, arising after exclusion of one or several supports from the design scheme, is determined by the dependence: $T_{D}=2 T_{S B}-T_{S A}, T_{S B}, T_{S A}$ is the forces of the constant stiffness system obtained by static calculations of the system with remote support and the intact system. At this stage, the set limits are verified and the value of $R$ target function is calculated.

2.3 Verification of system reliability index, calculation of failure and potential material damage probability. The reliability index is calculated in accordance with the theory outlined in [17]. Potential damage is estimated as the cost of the structure and other structures that may fail in case of its damage. The risk of material losses is calculated in accordance with the reliability theory [1] as the product of the failure probability multiplied by the amount of damage in monetary terms. The condition (1) associated with the relative risk allows screening off the solutions with "potentially dangerous" strength and rigidity 
reserves, which is an extraordinary approach that with a single-criteria solution searching scheme takes into account the structure optimality also from the safety point of view.

2.4 Forming set (II), including the best solutions. We will use the elitism strategy known in the evolutionary modeling theory [1]: the object version is placed to the set, if there is no object copy along the vector $\widetilde{\vec{P}}_{1}$ and when $R_{\max }^{(I I)}>\widetilde{R}$. Here $R_{\max }^{(I I)}$ is the

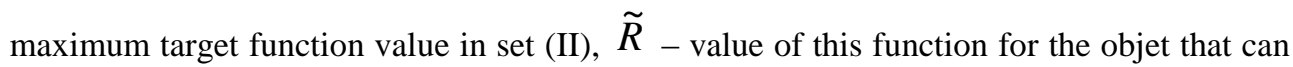
be added to the set.

Verifying the condition of iteration process interruption. If in set (II) for an empirically established whole number of appeals to cl. 2.4. This number depends on the number of variable parameters and the number of values of these parameters. To solve tasks of small computational capacity, this number can be taken equal to $80-100$.

\section{Results}

The parametric synthesis of a beam is considered, which design scheme is shown in Fig. 2 . It is believed that prefabricated reinforced concrete slabs pass the load on the beam both sides, and under the ceiling in each span of the beam, process equipment is located with a conventional cost of $10^{5}$ units. This cost is calculated when tacking into account the loss in case of damage to this equipment. The following values was assigned: $q=60 \mathrm{kN} / \mathrm{m}$, $l_{1}=l_{2}=3 \mathrm{~m}$. The beam is separated uniformly at a spacing of $0.5 \mathrm{~m}$ longwise by sections $s_{1}-s_{11}$ in which, during the search for a solution, internal forces are determined and mechanical characteristics are recalculated. Two emergency actions are considered. The first one is related to fast removal of the support $B$, second - removal of the support $C$. Deflection limits were assigned equal $[f]=0,21 \mathrm{~m}$.

Define discrete sets of variable parameters, MPa: for transverse reinforcement bars $\left\{y_{a}\right\}_{I, p}=\{240 ; 300\} \quad\left\{y_{a}\right\}_{I I, p}=\{170 ; 215\}$, for lengthwise reinforcement bars $\left\{y_{a}\right\}_{I, l}=\{240 ; 300 ; 400\},\left\{y_{a}\right\}_{I I, l}=\{270 ; 355 ; 435\}$. Where I,II - numbers of limit-state groups. Set: $\left\{D_{a}\right\}=\left\{(0,6,8,10,12)_{R s 1=240},(0,6,8,10,12,14)_{R s 2=300},(0,10,12,14,16,18)_{R_{s}=500}\right\}$ $\mathrm{mm}$.
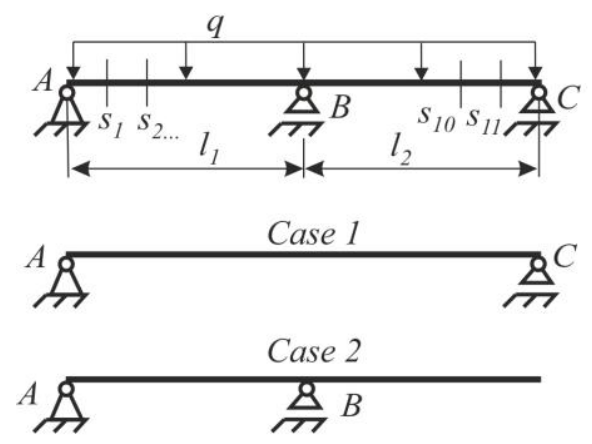

a)

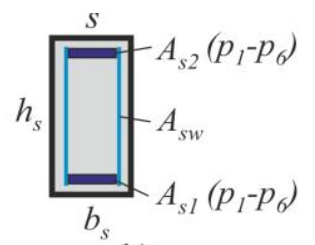

b)

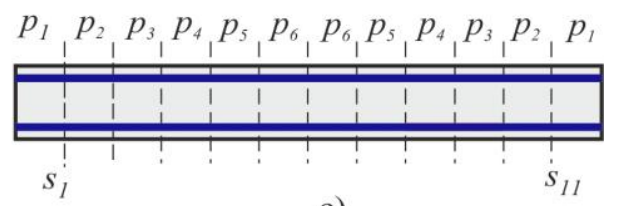

c)

Fig.2. Beam load diagram and local damage scenario (a); cross-sectional reinforcement scheme (b); grouping of reinforcement arrangement on sections $p_{1}-p_{6}$ (c) 
For concrete of the class B15, B20, B25, B30 the discrete sets of parameters can be written:

$$
\begin{array}{r}
\left\{y_{b}\right\}_{I I}=\{(11 ; 1,1),(15 ; 1,35),(18 ; 1,55),(22 ; 1,75)\}, \\
\left\{y_{b}\right\}_{I}=\{(8,5 ; 0,75),(11,5 ; 0,9),(14,5 ; 1,05),(17 ; 1,15)\} .
\end{array}
$$

The step of transverse reinforcement is considered fixed, taking it equal to $150 \mathrm{~mm}$ on the supporting sections, $750 \mathrm{~mm}$ and $300 \mathrm{~mm}$ long in the span areas. The concrete protective layer at the top and at the bottom of the beam is taken equal $25 \mathrm{~mm}$. Such combinations of cross-sectional sizes taken by length as the same, varied, cm: $\left\{G_{b}\right\}_{\text {rect }}=\left\{(300,200)_{1},(400,200)_{2},(450,200)_{3},(500,250)_{4}\right\}$. Concrete classes, diameters and classes of the reinforcement also varied. The grouping of parameters according to the symmetry of the reinforcement diameters is presented in fig. 1 , c.

Here the sections $p_{1}-p_{6}$ are introduced to identify the presence or absence of the reinforcement based on calculation. In general, 10 parameters varied, six of which are diameters of longitudinal reinforcement on sections $p_{1}-p_{6}$, concrete class, longitudinal reinforcement class, transversal reinforcement diameters and cross-section sizes. Security level is set $\Omega_{l}=1$, that is, the potential material damage in an accidental situation must not exceed the cost of the beam. The final solution is represented taking into account the unification of reinforcements. At each iteration, 25 structure designs were considered. After passing no more than 80 search algorithm iterations, solutions were obtained that satisfy the above constraints. Fig. 3 shows that without considering the risks of emergencies and ensuring the vitality (collapse resistance), the project has the least safety.

\section{Discussion}

It must be noted that when designing the structures using traditional approaches, the solutions are obtained lower in cost, but less safety in the context of potential emergency risks. The same conclusion can be made regarding the optimal design algorithms [18-21], where the cost expression of the risk of material losses is not fully considered. In our opinion, the minimum cost criterion without any consideration of material loss risks, cannot be the only means of obtaining optimal and safe solutions. 

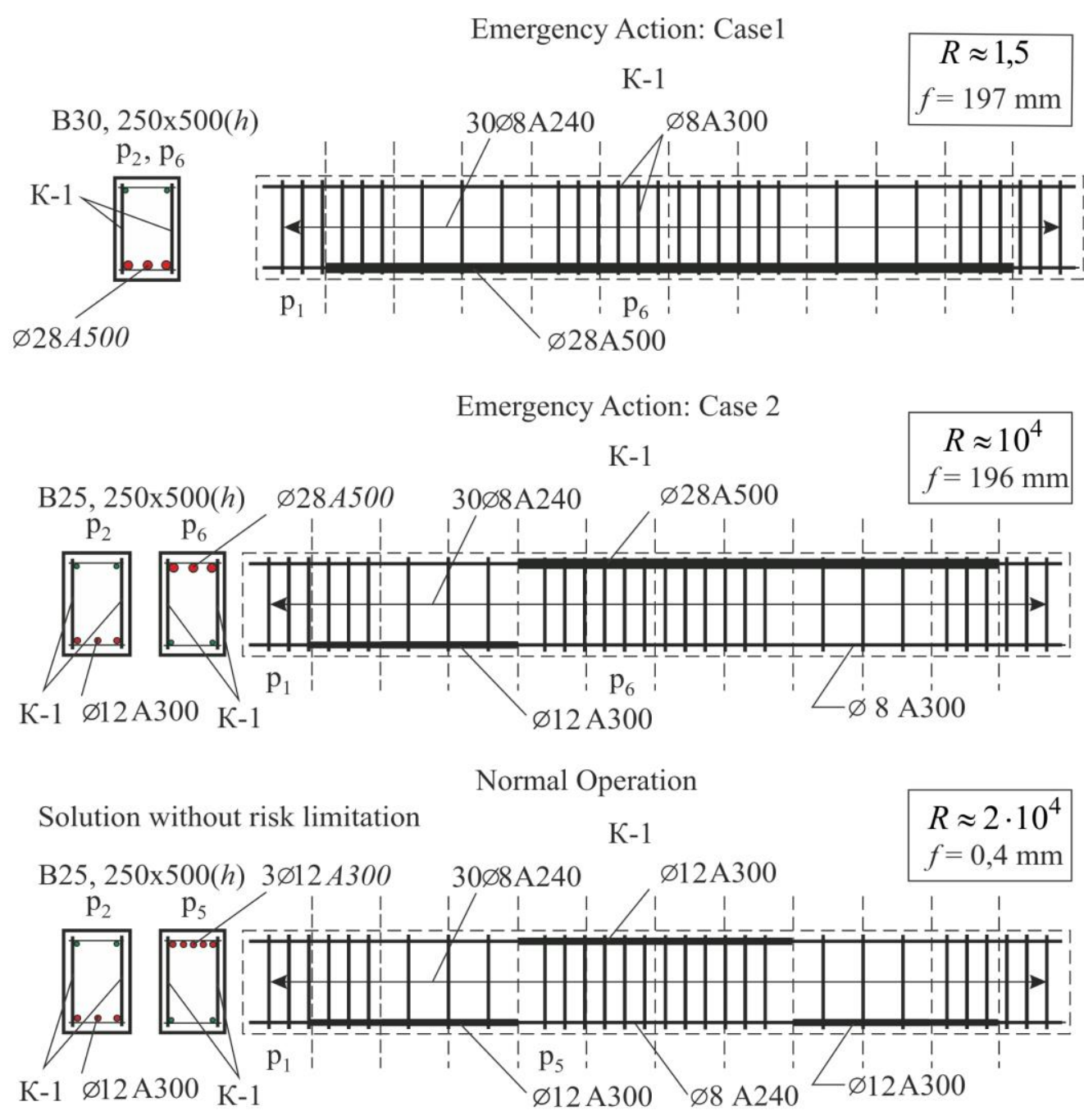

Final Solution

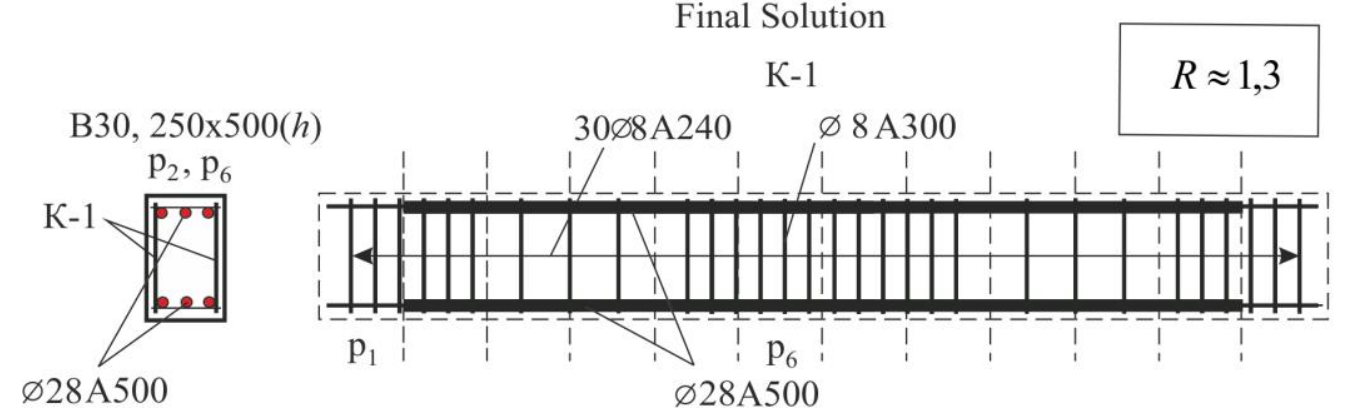

Fig.3. Search results and their comparison on the relative risk level 


\section{Conclusion}

A method for the optimal design of reinforced concrete beams with regard to their initial design reliability and safety based on the adapted genetic algorithm has been developed. An approach has been used, which includes the criteria of convergence to the specified safety level. The safety level is defined as the ratio of the cost terms of the material losses risk in an accidental situation to the initial cost of the object. Based on the example of a two-span reinforced concrete beam optimization, it has been shown that a comprehensive consideration of structure resistance in various local damage schemes allows improving the safety of using such structures significantly.

\section{References}

1. Tamrazyan A G 2011 Vestnik MGSU 2 pp 77-83.

2. Salmasi A C, Sheidaii M R 2017 Int. J. of Steel Structures 17(2) pp 543-551

3. Amiri S, Safari H, Mashhadi J 2018 Engineering Failure Analysis 84 pp 300-310

4. Travush V I, Fedorova N V Magazine of Civ. Eng. 20185 (81) pp 73-80

5. Serpik I N, Alekseytsev A V 2013 Magazine of Civ Eng. 9 (44) pp 23-29

6. Kuhlmann U, Roelle L, Izzuddin B A 2012 Struct. Eng. International 1(22) pp 86-92

7. Tamrazyan A G, Avetisyan L A 2018 IOP Conf. Ser.: Mater. Sci. Eng. 365052034

8. Tamrazyan A G, IOP Conf. Series: Materials Science and Engineering, 365, (2018) 052021

9. Tamrazyan A G, Popov D 2017 MATEC Web of Conferences 11700162

10. Shi L, Yang R-J, Ping Z 2013 Engineering Optimization 11(45) pp 1365-1377

11. Zhou H, Liang X, Ren X, Xie S-C 2014 J of Central South University 11 pp 4386-4394

12. Serpik I N, Alekseytsev A V, Balabin P Y 2017 Periodica Polytech. Civ. Eng. 61 pp $471-482$

13. Haupt R L, Haupt S E 2004 Practical genetic algorithms. New York: Wiley p 272

14. Alekseytsev A V 2013 Magazine of Civ. Eng. 5 (44) pp 28-37

15. Serpik I N, Alekseytsev A V, Balabin P Y, Kurchenko N S 2017 Magazine of Civ. Eng. 8 (76) pp 181-192

16. Alekseytsev A V, Akhremenko S A, 2018 Magazine of Civ. Eng. 5 (81), 32-42

17. Raizer V D 2009 Reliability of Structures: Analysis and Applications Backbone Publ. Co. $p 146$

18. Song X, Sun G, Li Q 2016 Thin-Walled Struct. 109 pp 132-142

19. Meng Z, Zhou H, Li G, Yang D 2016 Comp. \& Struct. 175 pp 65-73

20. Mella M A, Zio E 2016 Reliab. Eng. \& Sys. Saf. 152 pp 213-227

21. Zhang E, Chen Q 2016 Reliab. Eng. \& Sys. Saf. 145 pp 83-92 